\title{
A 15-GHz CMOS Multiphase Rotary Traveling-Wave Voltage-Controlled Oscillator
}

\author{
Changchun Zhang****, Zhigong Wang*, Yan Zhao*, and Sung Min Park**
}

\begin{abstract}
This paper presents a $15-\mathrm{GHz}$ multiphase rotary traveling-wave voltage-controlled oscillator (RTW VCO) where a shielded coplanar stripline (CPS) is exploited to provide better shielding protection and lower phase noise at a moderate cost of characteristic impedance and power consumption. Test chips were implemented in a standard 90-nm CMOS process, demonstrating the measured results

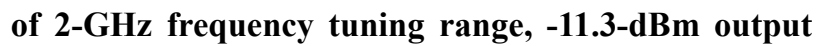
power, $-109.6-\mathrm{dBc} / \mathrm{Hz}$ phase noise at $1-\mathrm{MHz}$ offset, and 2-ps RMS clock jitter at $15 \mathrm{GHz}$. The chip core occupies the area of $0.2 \mathrm{~mm}^{2}$ and dissipates $12 \mathrm{~mW}$ from a single 1.2-V supply.
\end{abstract}

Index Terms-CMOS, shielded coplanar stripline, phase noise, rotary traveling-wave, VCO

\section{INTRODUCTION}

Voltage-controlled oscillators (VCOs) are categorized into three distinct types by their physical mechanism: RC-based VCOs, LC-based VCOs, and transmission line (TL)-based (or wave-based) VCOs. Among these types, the RC-based VCOs that include relaxation VCOs and ring VCOs exhibit the most inferior phase noise characteristics because of their inevitable resistor-related thermal noises. The LC-based VCOs are less competent than the TL-based VCOs because the feasible frequency range of the lumped passive inductors is much lower than that of the transmission-lines because of their parasitic

Manuscript received Sep. 8, 2011; revised Feb. 6, 2012.

* Southeast University, Nanjing, China

** Ewha Womans University, Seoul, Korea.

E-mail : changchunzhang2007@hotmail.com,smpark@ewha.ac.kr capacitances. In other words, the inherent parasitic capacitances of the TL-based VCOs can be almost absorbed into the transmission-lines, and thus the oscillation frequency can occur at a much higher frequency than other types.

Typically, three types of TL-based VCOs exist such as standing-wave VCOs, traveling-wave VCOs, and rotary traveling-wave (RTW) VCOs [1]. The standing-wave VCOs are unable to generate multiphase clocks and the travelling-wave VCOs show inferior phase noise characteristics due to their termination resistors. Thus, the RTW VCOs provide the best performance owing to their ability to generate multiphase clock outputs and demonstrate excellent phase noise characteristics [2-5]. In addition, a multiple array of RTW VCOs can be utilized as a clocking distribution circuit [2].

This paper presents a $15-\mathrm{GHz}$ RTW VCO which is realized in a standard 90-nm CMOS technology. Particularly, it employs half-quadrature topology and improved coupled coplanar waveguides so that the measured results demonstrate wider tuning range and lower power consumption with comparable phase noise than recently reported multiphase CMOS VCOs.

Section II and Section III provide the design

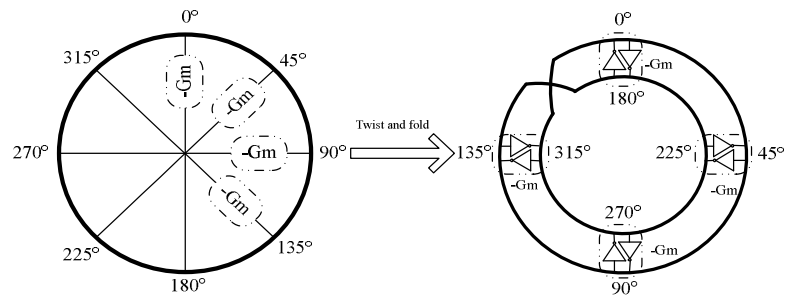

Fig. 1. RTW VCO topologies (a) untwisted single ring, (b) möbius twisted ring. 
methodology and the circuit description. Section IV demonstrates the chip implementation and its measured results. Conclusion follows in Section V.

\section{DeSign MeThodology}

Fig. 1 shows a conventional ring RTW VCO and its transformed version which is a Möbius ring RTW VCO. This transformation provides a number of advantages, including easier placement of $-\mathrm{Gm}$ cells, simpler tapping-off of the required differential clocks, better matching of differential clocks, less EMI radiation, better tolerance of large EMI, smaller physical area, etc.

Fig. 2 illustrates an equivalent transforming process of a periodically loaded coupled transmission line (PLCTL), where the parameters $L, R, C$, and $G$ indicate per-unitlength inductance, resistance, capacitance, and conductance, respectively. Also, 'unload', 'load', or 'PLCTL' represents unloaded CTL, load network, and PLCTL, respectively. Fig. 2(b) shows its lumped-element equivalent circuit of the PLCTL, and Fig. 2(c) depicts the odd-mode equivalent circuit by omitting the even-mode parts. Thereby, the relations of the circuit parameters between the equivalent circuit and the original one are clearly observed.

Table 1 lists the corresponding parameters and the equations related to both unloaded CTL and PLCTL, where ' $\mathrm{o}$ ' and ' $\mathrm{e}$ ' indicate the odd-mode and the evenmode, respectively. Also, the parameters $k_{\mathrm{L}}, Z, \gamma, \alpha, \beta, v$, and $l$ represent the inductance coupling coefficient, the characteristic impedance, the propagation constant, the attenuation constant, the phase constant, the phase velocity, and the segment length, respectively.

Unlike a TL with infinite-bandwidth, the PLCTL has passband-stopband characteristics due to its existing periodical discontinuity, indicating that a cutoff frequency exists for the desired low-pass transmission [6]. This frequency is called 'Bragg frequency', which is a notable effect on the characteristics of the PLCTL.

$$
f_{\text {bragg }}=1 / \pi l \sqrt{L_{\mathrm{PLCTL}} \cdot C_{\mathrm{PLCTL}}} .
$$

Fig. 3(a) shows an equivalent circuit of an RTW VCO where the load components (i.e. the combination of a cross-pair, varactors, and an output buffer) are represented as a passive load network with a negative conductance in parallel. Namely, the RTW VCO can be simplified to a combination of a passive PLCTL ring and multiple active energy-compensation cells. Then, this modeling is similar to that of an LC VCO in Fig. 3(b) because the passive PLCTL functions as a tank-load of the RTW VCO, as shown in Fig. 3(c).

The start-up conditions of the closed-loop RTW VCO can be obtained as below,

$$
\begin{gathered}
g_{m} \geq \frac{2}{Z_{\mathrm{PLCTLO}}} \exp \left(\alpha_{\mathrm{PLCTL}, \mathrm{O}} \cdot l\right) \\
\beta_{\text {PLCTL,O}} \cdot l=\frac{n \cdot \pi}{N}
\end{gathered}
$$

where $n$ is an arbitrary odd integer for the odd-mode operation of the RTW VCO [4].

Meanwhile, the fundamental oscillation frequency $\left(f_{\mathrm{RTW}}\right)$ of the RTW VCO is given by,

$$
f_{\mathrm{RTW}}=\frac{v_{\mathrm{PLCTL}, \mathrm{O}}}{2 N \cdot l}=\frac{1}{2 N l \sqrt{L_{\mathrm{PLCTL}, \mathrm{O}} C_{\mathrm{PLCTL}, \mathrm{O}}}} .
$$

Then, the tuning range of the RTW VCO is given by,

$$
\frac{1}{2 N l \sqrt{L_{\mathrm{PLCTL}, O} C_{\mathrm{PLCTL}, \mathrm{O}, \max }}} \leq f_{0} \leq \frac{1}{2 N l \sqrt{L_{\mathrm{PLCTL}, \mathrm{O}} C_{\mathrm{PLCTL}, \mathrm{O} \text { min }}}} .
$$

Also, the power dissipation of the RTW VCO is given by,

$$
P_{\text {diss }}=\frac{V_{\mathrm{DD}}^{2}}{\mathrm{Z}_{\mathrm{PLCTL}, \mathrm{O}}^{2}} \cdot R_{\text {loop }}
$$

where $R_{\text {loop }}$ is the loop resistance $[2,7]$.

The phase noise of the RTW VCO can be derived as below, as for an LC VCO in Ref. [8]:

$$
\begin{aligned}
& L\{\Delta \omega\}=\frac{2 D}{(\Delta \omega)^{2}+(D)^{2}} \approx \frac{2 D}{(\Delta \omega)^{2}} \\
& \text { Noise-to- } \\
& \text { carrier ratio } \\
& D \sim\left(\frac{\omega_{0}}{Q}\right) \cdot\left(\frac{1}{V_{0}^{2}} \cdot \frac{k_{B} T}{C} \cdot \frac{Q}{Q_{\text {loaded }}}\right)=\overbrace{\frac{1}{V_{0}^{2}} \cdot\left(\frac{k_{B} T}{C}\right)} \cdot\left(\frac{\omega_{0}}{Q_{\text {loaded }}}\right) \\
& \text { Loss } \\
& \text { (unloaded) Linewidth } \\
& \text { Sensitivity } \begin{array}{c}
\text { Loss } \\
\text { (loaded) }
\end{array}
\end{aligned}
$$




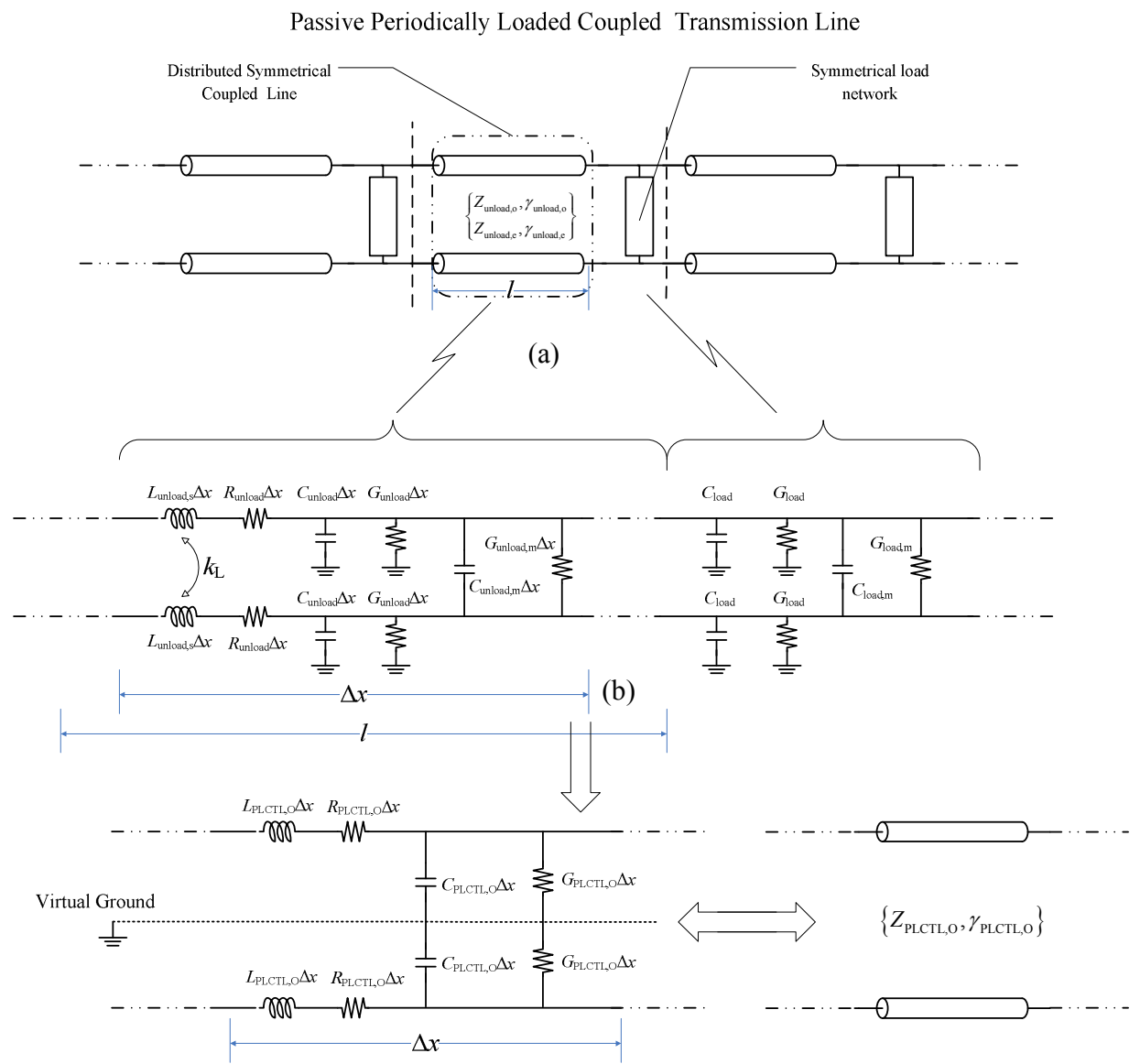

(c)

Fig. 2. (a) Segments of a passive PLCTL, (b) its lumped-element equivalent circuit, (c) its odd-mode equivalent circuit.

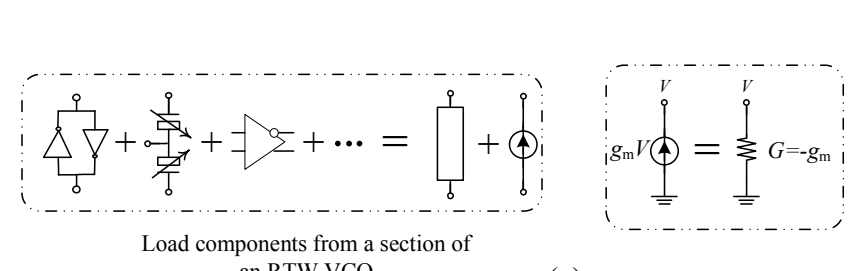
an RTW VCO

(a)

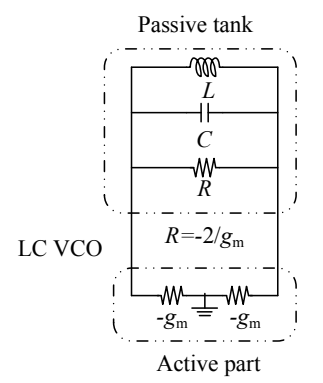

(b)

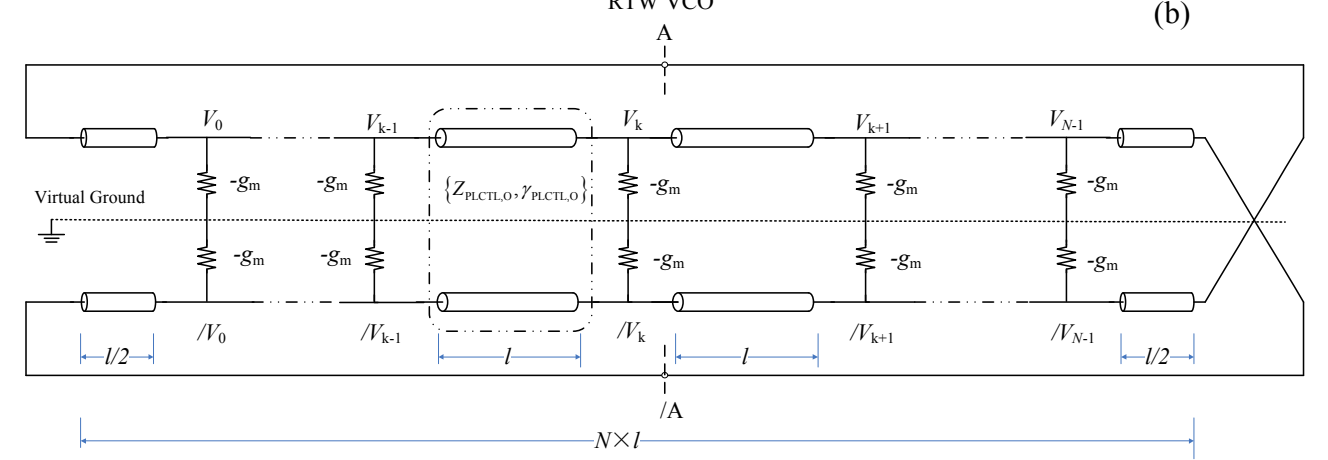

(c)

Fig. 3. Equivalent circuits of (a) load components from a section of an RTW VCO, (b) a differential LC VCO, (c) an RTW VCO. 
Table 1. Parameters and equations related to unloaded CTL and PLCTL

\begin{tabular}{|c|c|c|c|}
\hline & unloaded CTL & PLCTL & \multirow{13}{*}{$\begin{array}{l}\gamma=\alpha+j \beta \\
(R<<\omega L, \text { and } G<<\omega C) \\
\left.\text { (for PLCTL, } \omega<<2 \pi f_{\text {bragg }}\right) \\
Z \approx \sqrt{L / C} \\
\gamma \approx \frac{1}{2}\left(\frac{R}{Z}+G Z\right)+j \omega \sqrt{L C} \\
v \approx 1 / \sqrt{L C} \\
Q \approx \frac{\omega \sqrt{L C}}{(R / Z+G Z)}=\frac{1}{R / \omega L+G / \omega C}\end{array}$} \\
\hline$L_{o}$ & $L_{\text {unload, },}\left(1-k_{\mathrm{L}}\right)$ & $L_{\text {unload, }, s}\left(1-k_{\mathrm{L}}\right)$ & \\
\hline$R_{o}$ & $R_{\text {unload }}$ & $R_{\text {unload }}$ & \\
\hline$C_{o}$ & $C_{\text {unload }}+2 C_{\text {unload,m }}$ & $\begin{array}{l}C_{\text {unload }}+2 C_{\text {unload, } \mathrm{m}}+ \\
C_{\text {load }} / l+2 C_{\text {load, }} / l\end{array}$ & \\
\hline$G_{o}$ & $G_{\text {unload }}+2 G_{\text {unload,m }}$ & $\begin{array}{c}G_{\text {unload }}+2 G_{\text {unload,m }}+ \\
G_{\text {load }} / l+2 \mathrm{G}_{\text {load,m }} / l\end{array}$ & \\
\hline$L_{e}$ & $L_{\text {unload }, s}\left(1+k_{\mathrm{L}}\right)$ & $L_{\text {unload }, s}\left(1+k_{\mathrm{L}}\right)$ & \\
\hline$R_{e}$ & $R_{\text {unload }}$ & $R_{\text {unload }}$ & \\
\hline$C_{e}$ & $C_{\text {unload }}$ & $C_{\text {unload }}+C_{\text {load }} / l$ & \\
\hline$G_{e}$ & $G_{\text {unload }}$ & $G_{\text {unload }}+G_{\text {load }} / l$ & \\
\hline$Z$ & \multicolumn{2}{|c|}{$\sqrt{(j \omega L+R) /(j \omega C+G)}$} & \\
\hline$\gamma$ & \multicolumn{2}{|c|}{$\sqrt{(j \omega L+R) \cdot(j \omega C+G)}$} & \\
\hline$v$ & \multicolumn{2}{|c|}{$\omega / \beta$} & \\
\hline$Q$ & \multicolumn{2}{|c|}{$\beta / 2 \alpha$} & \\
\hline
\end{tabular}

where $D, k_{\mathrm{B}}, T, Q, Q_{\text {loaded }}, V_{0}, \omega_{0}$, and $C$ represent the diffusion constant, the Boltzmann constant, the absolute temperature, the unloaded quality-factor of the tank, the loaded quality-factor of the tank, the oscillation amplitude, the oscillation frequency, and the tank capacitance, respectively.

The optimization of the phase noise requires two-step procedures. The first is to select a high-Q loaded resonator tank, and the second is to achieve the highest possible line-width compression ratio which involves the loaded $\mathrm{Q}$ and the noise-to-carrier ratio.

Namely, an RTW VCO can achieve an excellent phase noise performance only with high loaded-Q factor in the selected transmission lines. Yet, another design tradeoff exists between power consumption and phase noise.

Due to the Bragg frequency, the Q-factor $\left(Q_{\text {PLCTL }}\right)$ of the passive PLCTL is given by [3],

$$
Q_{\mathrm{PLCTL}}=\underbrace{\frac{1}{R_{\mathrm{PLCTL}} / \omega L_{\mathrm{PLCTL}}+G_{\mathrm{PLCTL}} / \omega C_{\mathrm{PLCTL}}}}_{\begin{array}{c}
\text { from } \\
\text { resistive loss }
\end{array}} \cdot(\underbrace{\left.1-\frac{f^{2}}{f_{\text {bragg }}^{2}}\right)}_{\begin{array}{c}
\text { from } \\
\text { reflection loss }
\end{array}}
$$

where $Q_{\text {PLCTL }}$ depends not only on resistive loss, but also on reflection loss.

The resistive loss attenuates signals and gives rise to thermal noise, while the reflection loss reduces the effective signal transmission via reflection. For $f<<f_{\text {bragg, }}$, the $Q_{\text {PLCTL }}$ becomes dependent upon resistive loss only. Then, it reduces to $1 /\left(R_{\mathrm{PLCTL}} / \omega L_{\mathrm{PLCTL}}+G_{\mathrm{PLCTL}} / \omega C_{\mathrm{PLCTL}}\right)$. As $f$ approaches $f_{\text {bragg, }}$ the reflection loss gradually becomes dominant over the resistive loss.

Hence, the loaded Q ( $\left.Q_{\text {loaded }}\right)$ of an RTW VCO depends not only on the resistive loss, but also upon the reflection loss. Particularly, the latter is closely related to the interval between two neighboring discontinuities. Thereby, the shorter the interval is, the higher the Bragg frequency becomes, the better the quality factor is, and the steeper the clock waveforms become.

Meanwhile, the number $(n)$ of the sustained harmonics of the PLCTL (correspondingly, of the RTW VCO) must meet the condition below:

$$
(2 n+1) f_{\mathrm{RTW}}<f_{\text {bragg }} \Rightarrow n<\frac{1}{2} \cdot\left(\frac{f_{\text {bragg }}}{f_{\mathrm{RTW}}}-1\right)
$$

where the relation between $n$ and $N$ is given by,

$$
n=\left\lfloor\frac{N}{\pi}-\frac{1}{2}\right\rfloor .
$$

With the total gain of $N \cdot g_{\mathrm{m}}$ unchanged, a larger $N$ results in a higher Bragg frequency, which in turn leads to a higher Q-factor of the loaded CTL and steeper waveform edges. Eventually, these higher Q-factor and steeper edges improve the phase noise. However, too large $N$ degrades the phase noise because of the serious high-frequency loss from the PLCTL and of the reduced Q-factor from the drastic increase of small-size gate resistance. Thus, a compromise must occur between the Q-factor and the number of gain stages which loads the transmission lines. 
Also, (7) indicates that increasing capacitance (which is equivalent to the increase of the tank energy) results in lower phase noise at the expense of higher power dissipation, provided that the amplitude $(A)$ is kept constant (e.g. at the border of voltage-limited region). It is because a larger capacitance corresponds to a lower characteristic impedance of the PLCTL tank, and thus leads to lower phase noise. Yet, the characteristic impedance should not be too much lowered since the condition below must be satisfied [2]:

$$
Z_{\text {PLCTL }}>\frac{R_{\text {loop }}}{2} .
$$

\section{CirCUIT DESCRIPTION}

Fig. 4 depicts the three-dimensional layout of the proposed RTW VCO, which consists of the proposed shielded coplanar stripline, eight $-\mathrm{Gm}$ cells (which comprises inverter cross-pairs and varactors), and four output buffers. In particular, the oscillation tank comprises the shielded CPS, varactors, and the parasitic capacitances from inverters and output buffers.

\section{Proposed Shielded Coplanar Stripline}

Typically, there are four types of symmetric coupled lines: (a) coplanar stripline (CPS) [3, 4], (b) coupled microstrip line [9], (c) coupled coplanar waveguide (CCPW), and (d) folded coupled coplanar waveguide (FC_CPW) in which an U-shaped return ground is employed [10]. Even in a symmetric coupled line, electromagnetic interference (EMI) effect would become very serious both at high oscillation frequencies and with full swing square waveforms (which are commonly occurred at RTW VCOs). Therefore, a design tradeoff exists between shielding and characteristic impedance $\left(Z_{\mathrm{o}}\right)$. However, the former is related to both EMI and substrate loss, and the latter is closely related to power consumption in (5). Thus, better shielding indicates larger capacitance and smaller inductance, which will induce lower $Z_{\mathrm{o}}$ and hence higher power consumption. Yet, this lower $Z_{\mathrm{o}}$ benefits the phase noise of the RTW VCO, as described in Section II. Among the forementioned four CTLs, the CPS yields the highest $Z_{\mathrm{o}}$ and the poorest shielding, whereas the FC_CPW provides the

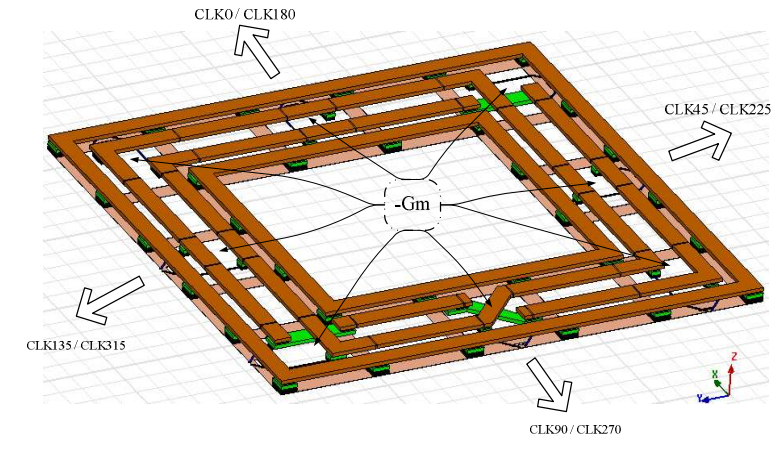

Fig. 4. Three-dimensional layout of the RTW VCO topology.

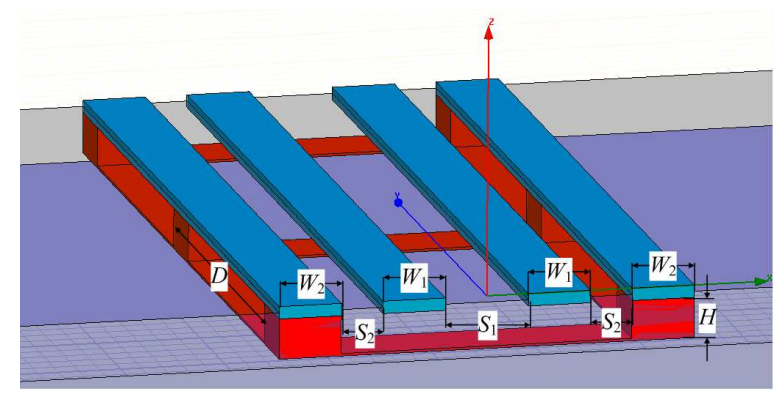

(a)

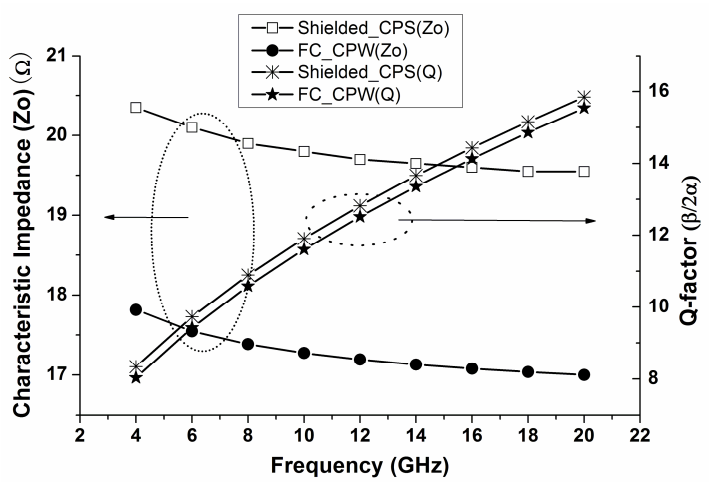

(b)

Fig. 5. (a) Three-dimensional layouts of the shielded CPS, (b) their comparison to FC_CPW in terms of odd-mode characteristic impedance and Q-factor.

lowest $Z_{\mathrm{o}}$ and the most excellent shielding.

Fig. 5(a) illustrates the proposed shielded CPS which is an optimized version between a CPS (or CCPW) and a FC_CPW. The performance of the shielded CPS can be easily controlled by either altering the ground grid density or varying the distance between the shielding ground and signal stripes. Fig. 5(b) depicts the simulation results of both the shielded CPS and the FC_CPW for the odd-mode $Z_{\mathrm{o}}$ and the Q-factor, in which the shielded CPS possesses a higher $Z_{O}$ with a slightly better $Q$-factor as the representative FC CPW. Also, the Q-factor of the 
shielded CPS increases as the operation frequency, which is easily predicted from the definition of $Q \approx$ $1 /(R / \omega L+G / \omega C)$ in Table 1.

Yet, as either the distance $\left(S_{1}\right)$ between two signal strips or the distance $\left(S_{2}\right)$ between signal strip and ground becomes larger, the odd-mode inductance increases, the capacitance reduces, and the proximity effect weakens. Therefore, both the odd-mode $Z_{\mathrm{o}}$ and the Q-factor increase at the cost of three factors: (a) EMI becomes more severe for a larger $S_{1}$ or $S_{2}$, (b) the matching between differential signals worsens, and (c) the chip area becomes larger. If the width $(W)$ of the signal strip is wide enough, the odd-mode Q-factor increases and the odd-mode $Z_{\mathrm{o}}$ decreases.

\section{Varactors}

Frequency tuning is conducted by varying either the ring-length, or the effective dielectric constant, or the effective capacitance [21], among which the effective capacitance tuning with varactors is most popular due to its easiness and compactness. Particularly, accumulationmode NMOS varactors provide excellent performance [22].

It is seen from (4) that the tuning range $\left(\Delta f_{\mathrm{o}}\right)$ of an RTW VCO is proportional to the ratio of $C_{\mathrm{tank}, \mathrm{O} \text {, max }} /$ $C_{\text {tank,O,min }}$. Here, $C_{\text {tank,O }}$ is the sum of the equivalent oddmode unit-length capacitance from the distributed tank, i.e. $C_{\mathrm{tank}, \mathrm{O}}=C_{\mathrm{CPS}, \mathrm{O}}+\left(C_{-G \mathrm{~m}, \mathrm{O}}+C_{\mathrm{bur}, \mathrm{O}}+C_{v a r, \mathrm{O}}\right) / l . C_{\mathrm{CPS}, \mathrm{O}}$ is the equivalent unit-length capacitance from the shielded CPS, and $C_{-G \mathrm{~m}, \mathrm{O}} / l, C_{\mathrm{buf}, \mathrm{O}} / l, \& C_{v a r, \mathrm{O}} / l$ represent the unit-length capacitance from -Gm cells, output buffers, and varactors, respectively. Therefore, $C_{v a r, O}$ should be much larger than other three capacitances for a wide tuning range.

Now, the Q-factor $\left(Q_{\text {tank }}\right)$ of the distributed tank in the RTW VCO is given by,

$$
\begin{gathered}
Q_{\mathrm{tank}}=Q \cdot\left(1-\frac{f^{2}}{f_{\text {bragg }}^{2}}\right) \\
\frac{1}{Q}=\frac{1}{Q_{L}}+\frac{1}{Q_{C}} \\
\frac{1}{Q_{C}}=\sum_{i} \frac{1}{Q_{i} \cdot\left(\frac{C}{C_{i}}\right)}
\end{gathered}
$$

where $Q_{\mathrm{L}}$ is the Q-factor of the unit-length equivalent

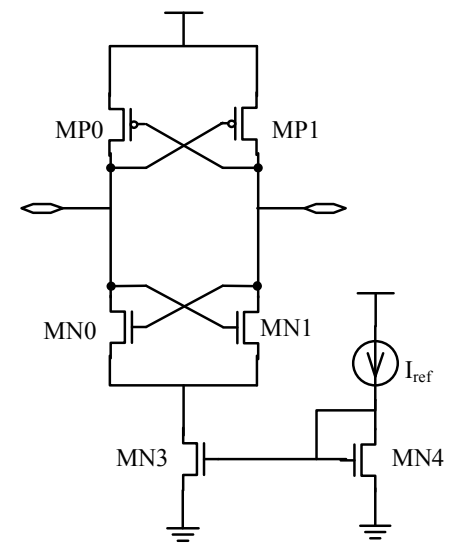

Fig. 6. Schematic diagram of a gain cell.

inductance of the distributed tank, $C \& Q_{\mathrm{C}}$ are the total unit-length equivalent capacitance and the Q-factor of the tank, respectively. Also, $C_{i} \& Q_{i}$ represent $C_{\mathrm{CPS}, \mathrm{O}}, C_{\text {. }}$ ${ }_{G \mathrm{~m}, \mathrm{O}} / l, C_{\mathrm{buf}, \mathrm{O}} / l$, or $C_{\mathrm{var}, \mathrm{O}} / l$, and their respective Q-factor.

Since the capacitance of the shielded CPS is equivalent to an MIM capacitance, its Q-factor is much higher than that of other capacitances. Also, $C_{-G \mathrm{~m}, O} / l$ and $C_{\text {buf }, O} / l$ show higher Q-factor than $C_{\text {var }, O} / l$. Thus, $Q_{\mathrm{C}}$ in (14) is mainly determined by $Q_{\text {var }}$ due to its lower Qfactor and bigger capacitance. Besides, $Q_{\mathrm{L}}$ is much higher than $Q_{\text {var }}$ and then the tank Q-factor $\left(Q_{\text {tank }}\right)$ is dominated by $Q_{\text {var }}$.

In this work, the minimum Q-factor of the varactors is only 6 at $15 \mathrm{GHz}$ while the Q-factor of the proposed shielded CPS reaches 14 .

\section{Gain Cell and Output Buffers}

Fig. 6 presents the schematic diagram of a gain stage, where the complementary configuration yields a higher transconductance, more symmetric oscillation waveforms, and more compact layout [14]. Here, the wave rotation uncertainty may occur and thus the direction control transistors with minimum size are added [5]. Fig. 7 illustrates the proposed $-G_{\mathrm{m}}$ cell which consists of a pair of cross-coupled CMOS inverters and a couple of varactors. The CMOS cross-coupled pair takes the same configuration as the gain stage in Fig. 6, except for the removed tail current source. Removing the tail current source provides a number of advantages [15-20]: (a) it eliminates the voltage overhead, thus maximizing the signal swing and improving the phase noise, (b) the gatewidths can be reduced because the transistors can be 


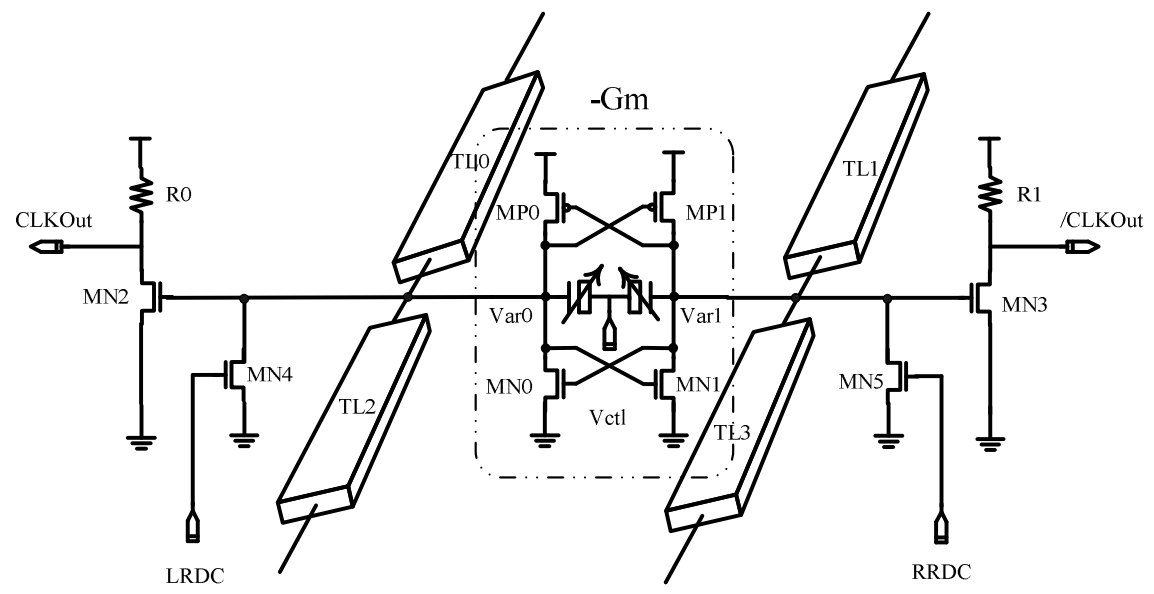

Fig. 7. $-G_{\mathrm{m}}$ cell with varactors, clock buffers, and direction control transistors.

biased at higher transconductance, and thus its nonlinear parasitic capacitances decrease accordingly. Typically, these parasitic capacitances are closely related with the tuning range and the noise conversion from both $\mathrm{CM}$ (common-mode) noise and AM-noise to phase noise. Besides, these parasitic capacitances degenerate the Qfactor of tank-load. (c) tail current-source is a notorious thermal noise and flicker noise source, and thus its removal improves the phase noise performance. Yet, frequency pushing problem occurs with no tail currentsource.

Meanwhile, resistor-loaded common-source output buffers are employed in this work for the sake of integration-level even at the cost of larger phase noise from the loaded resistors. The resistance value is judiciously chosen to be $100-\Omega$ in order to optimize the design tradeoff between gain and reflection.

\section{MEASUREMENT RESUltS}

The proposed RTW VCO has been fabricated in a standard 90-nm CMOS technology. Fig. 8 depicts the chip microphotograph, where the core occupies the area of $450 \mu \mathrm{m} \times 450 \mu \mathrm{m}$. DC measurements reveal that the power consumption is $12 \mathrm{~mW}$ in total from a single 1.2V supply.

For RF measurements, on-wafer probing has been conducted by utilizing an RF probe-station with a widebandwidth oscilloscope (Agilent 86100A Infinium DCA) and an E4440a digital spectrum analyzer.

Fig. 9 demonstrates the measured oscillation frequency and output power versus tuning voltage of the RTW VCO,

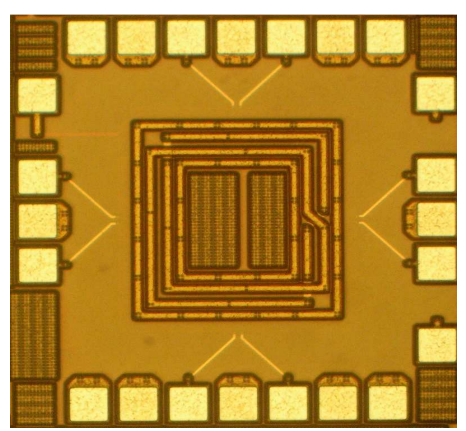

Fig. 8. Chip microphotograph of the proposed RTW VCO.

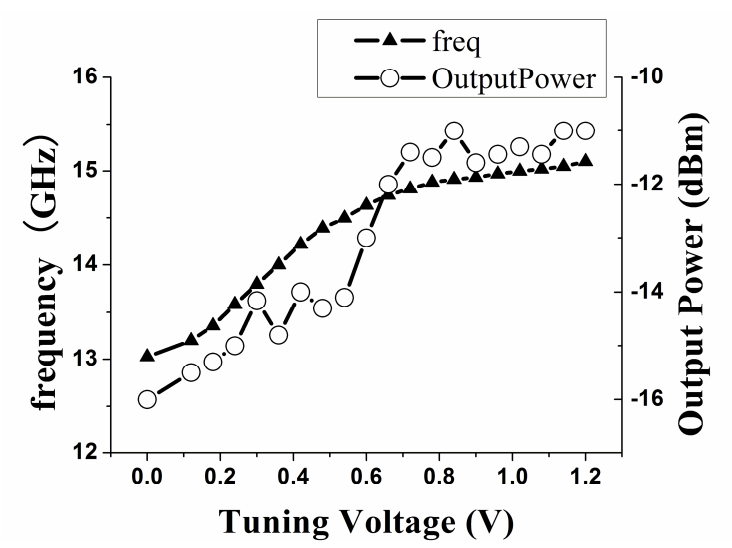

Fig. 9. Measured oscillation frequency and output power versus tuning voltage of the proposed RTW VCO.

achieving the tuning range of $2 \mathrm{GHz}$ and the corresponding $\mathrm{VCO}$ gain of $1.7 \mathrm{GHz} / \mathrm{V}$ on average.

Fig. 10 shows the measured waveforms of the two adjacent $15-\mathrm{GHz}$ clocks with $45^{\circ}$ phase offset, where each clock waveform has an RMS jitter of 2 ps and the amplitude of $167 \mathrm{mV}_{\mathrm{pp}}$. These results confirm the measurements of the frequency domain. 


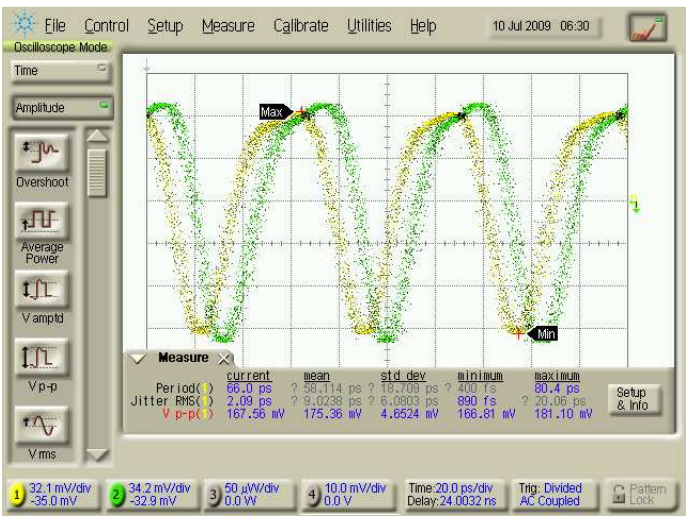

Fig. 10. Measured waveforms of the two adjacent clocks with $45^{\circ}$ phase offset.

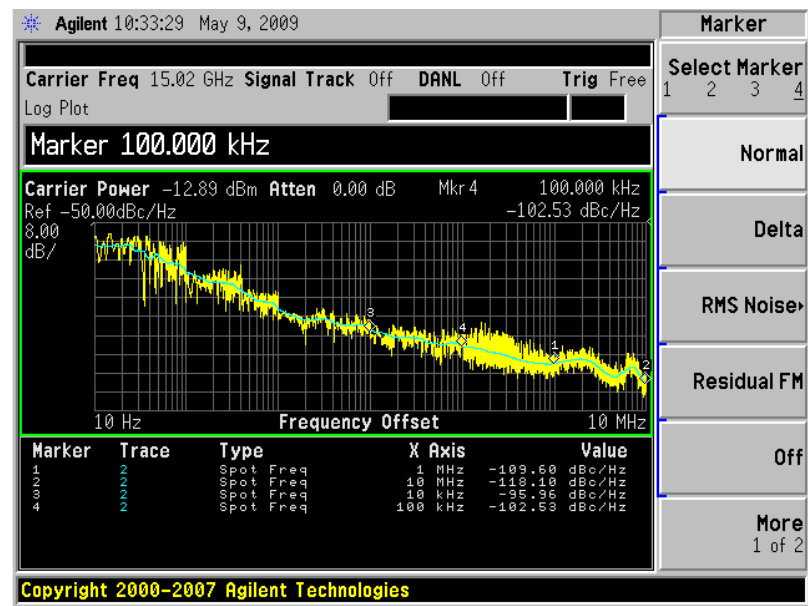

(a)

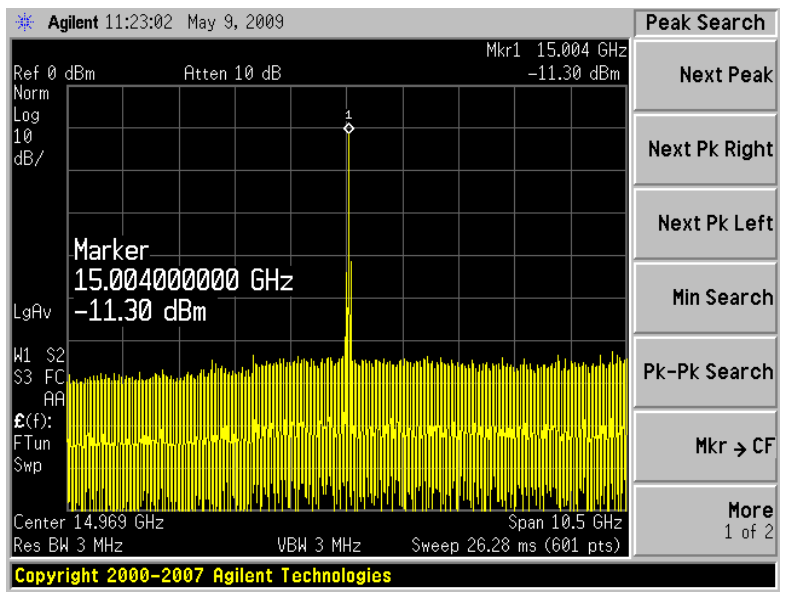

(b)

Fig. 11. (a) Measured phase noise of the RTW VCO, (b) the power spectrum of the generated $15-\mathrm{GHz}$ clock.

Fig. 11 shows the measured phase noise and the power spectrum of the RTW VCO at the center frequency of 15 $\mathrm{GHz}$, where the generated $15-\mathrm{GHz}$ clock achieves the power spectrum of $-11.3 \mathrm{dBm}$, and the phase noise of $95.96 \mathrm{dBc} / \mathrm{Hz}$ at $10-\mathrm{kHz}$ offset, $-102.5 \mathrm{dBc} / \mathrm{Hz}$ at $100-$ $\mathrm{kHz}$ offset, and $-109.6 \mathrm{dBc} / \mathrm{Hz}$ at $1-\mathrm{MHz}$ offset, respectively.

Table 2 summarizes the performance of the proposed RTW VCO along with the comparison with the prior arts, in which the performance is first evaluated by utilizing a typical figure-of-merit (FoM) defined by,

$$
F O M=L\left(\Delta f_{\text {offset }}\right)-20 \log \left(\frac{f_{0}}{\Delta f_{\text {offset }}}\right)+10 \log (P)
$$

where $L\left(\Delta f_{\text {offset }}\right), f_{0}$, and $P$ represent the measured phase noise at the offset frequency $\Delta f_{\text {offset }}$, the oscillation frequency, and the power consumption $(\mathrm{mW})$, respectively.

In addition, another figure-of-merit $\left(F o M_{T}\right)$ is proposed as below, because frequency tuning range is a crucial performance criterion in the performance evaluation.

$$
F O M_{T}=L\left(\Delta f_{\text {offset }}\right)-20 \log \left(\left(\frac{f_{0}}{\Delta f_{\text {offset }}}\right) \cdot\left(\frac{F T R}{10}\right)\right)+10 \log (P)
$$

where FTR is the percentage of frequency tuning range.

It is clearly seen in both FoM and $\mathrm{FoM}_{\mathrm{T}}$ that the proposed RTW VCO demonstrates superior performance among the recently published multiphase CMOS VCOs.

\section{Conclusions}

A $15-\mathrm{GHz}$ multiphase RTW $\mathrm{VCO}$ is realized in a standard 90-nm CMOS process with the core area of 450 $\mu \mathrm{m} \times 450 \mu \mathrm{m}$. Measured results demonstrate the frequency tuning range of $2 \mathrm{GHz}$, the output power of $11.3 \mathrm{dBm}$, and the phase noise of $-109.6 \mathrm{dBc} / \mathrm{Hz}$ at $1-$ $\mathrm{MHz}$ offset. Also, the clock waveforms show the RMS jitter of 2 ps with the amplitude of $167 \mathrm{mV}_{\mathrm{pp}}$. DC measurements reveal the power dissipation of $12 \mathrm{~mW}$ from a single 1.2-V supply. Conclusively, the proposed RTW VCO provides a low-power low-cost solution for the applications of high-speed digital interface and RF systems, such as clock and data recovery circuits and integrated phase arrays. 
Table 2. Performance summary of the RTW VCO and comparison with the prior arts of multiphase CMOS VCOs

\begin{tabular}{|c|c|c|c|c|c|c|c|c|}
\hline Parameters & {$[23]$} & {$[12]$} & {$[11]$} & {$[5]$} & {$[9]$} & {$[4]$} & {$[24]$} & This work \\
\hline CMOS tech. & $0.18 \mu \mathrm{m}$ & $0.18 \mu \mathrm{m}$ & $0.13 \mu \mathrm{m}$ & $90 \mathrm{~nm}$ & $0.13 \mu \mathrm{m}$ & $0.18 \mu \mathrm{m}$ & $90 \mathrm{~nm}$ & $\mathbf{9 0} \mathbf{n m}$ \\
\hline Frequency & $5 \mathrm{G} \mathrm{Hz}$ & $10 \mathrm{GHz}$ & $11 \mathrm{GHz}$ & $11 \mathrm{GHz}$ & $12 \mathrm{GHz}$ & $15 \mathrm{GHz}$ & $18 \mathrm{GHz}$ & $\mathbf{1 5 ~ G H z}$ \\
\hline Configuration & $\begin{array}{c}\text { Revised } \\
\text { coupled } \\
\text { VCO }\end{array}$ & $\begin{array}{c}\text { Revised } \\
\text { RTW VCO }\end{array}$ & $\begin{array}{c}\text { Revised } \\
\text { LC-tuned } \\
\text { ring VCO }\end{array}$ & RTW VCO & RTW VCO & RTW VCO & VCO array & RTW VCO \\
\hline No. of phases & Half- Quad & Half- Quad & Half- Quad & $24-$ Phase & Quad. & Half- Quad & Half- Quad. & Half-Quad. \\
\hline Tuning range & $\begin{array}{c}400 \mathrm{MHz} \\
(7.5 \%)\end{array}$ & $\begin{array}{c}1 \mathrm{GHz} \\
(10 \%)\end{array}$ & $\begin{array}{c}2.7 \mathrm{GHz} \\
(23 \%)\end{array}$ & $\begin{array}{c}1 \mathrm{GHz} \\
(9 \%)\end{array}$ & $\begin{array}{c}1.2 \mathrm{GHz} \\
(10 \%)^{*}\end{array}$ & $\begin{array}{c}250 \mathrm{MHz} \\
(1.7 \%)\end{array}$ & N/A & $\begin{array}{c}\mathbf{2 G H z} \\
(\mathbf{1 4 \%})\end{array}$ \\
\hline $\begin{array}{c}\text { Phase noise } \\
(\mathrm{dBc} / \mathrm{Hz} @ 1 \mathrm{MHz})\end{array}$ & -115.4 & -105 & -107 & -96.65 & -105 & -112.2 & -97.52 & $\mathbf{- 1 0 9 . 6}$ \\
\hline Power diss. $(\mathrm{mW})$ & 7.4 & N/A & 26 & 70 & 30 & 52 & 9.6 & $\mathbf{1 2}(\mathbf{1 0 m A})$ \\
\hline FoM $(\mathrm{dBc})$ & -181 & N/A & -174 & -159 & -172 & -179 & -173 & $\mathbf{- 1 8 2}$ \\
\hline FoM $\mathrm{T}(\mathrm{dBc})$ & -179 & N/A & -182 & -158 & $-172 *$ & -164 & N/A & $\mathbf{- 1 8 5}$ \\
\hline
\end{tabular}

*switch array technique adopted

\section{ACKNOWLEDGMENTS}

This work was supported by the National Research Foundation of Korea Grant funded by the Korean Government (No. 2012-0000699).

\section{REFERENCES}

[1] W. F. Andress, and D. Ham, "Recent developments in standing-wave oscillator design: Review," IEEE RFIC Symp., pp. 119-122, 2004.

[2] J. Wood, T. C. Edwards, and S. Lipa, "Rotary traveling-wave oscillator arrays: A new clock technology", IEEE J. of Solid-State Circuits, Vol. 36, No. 11, pp. 1654-1665, Nov. 2001.

[3] G. Le Grand de Mercey, “18GHz-36GHz rotary traveling wave voltage controlled oscillator in a CMOS technology", Ph.D. dissertation, Dept. Inform. Tech., Univ. Bundeswehr, Munich, Germany, Aug. 2004.

[4] H. H. Hsieh, Y. C. Hsu, and L. H. Lu, "A 15/30$\mathrm{GHz}$ dual-band multiphase voltage controlled oscillator in 0.18- $\mu \mathrm{m}$ CMOS", IEEE Trans. on Microwave Theory and Technique, Vol. 55, No. 33, pp. 474-483, Mar. 2007.

[5] N. Tzartzanis and W. W. Walker, "A reversible poly-phase distributed VCO”, IEEE Tech. Digest of ISSCC, pp. 596-597, Feb. 2006.

[6] R. E. Collin, 'Foundations for Microwave Engineering', 2 ${ }^{\text {nd }}$ Ed., Jonn Wilely\&Sons, NJ, 2001.

[7] Z. T. Yu and X. Liu, "Low-power rotary clock array design", IEEE Trans. on VLSI Systems, Vol.
15, No. 1, pp. 5-12, Jan. 2007.

[8] D. Ham and A. Hajimiri, "Virtual damping and einstein relation in oscillators," IEEE J. of Solid State Circuits, Vol. 38, No. 3, pp. 407-418, Mar. 2003.

[9] F. B. Abdeljelil, W. Tatinian, L. Carpineto, and G. Jacquemod, "Design of a CMOS $12 \mathrm{GHz}$ rotary traveling wave oscillator with switched capacitor tuning," IEEE RFIC Symp., pp. 579-582, 2009.

[10] N. Nedovic et al., "A 40-44 Gb/s 3×oversampling CMOS CDR/1:16 DEMUX," IEEE J. of SolidState Circuits, Vol. 42, No. 12, pp. 2726-2735, Dec. 2007.

[11] D. Axelrad et al., "A multi-phase $10 \mathrm{GHz} \mathrm{VCO}$ in CMOS/SOI for 40Gbit/s SONET OC-768 clock and data recovery circuits", IEEE RFIC Symp., pp. 573-576, 2005.

[12] J. Lee and B. Razavi, "A 40-Gb/s clock and data recovery circuit in $0.18-\mu \mathrm{m}$ CMOS technology," IEEE J. of Solid-State Circuits, Vol. 38, No. 12, pp. 2181-2190, Dec. 2003.

[13] J. C. Chien, and L. H. Lu, “A 32-GHz rotary traveling-wave voltage controlled oscillator in

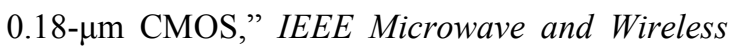
Components Letters, Vol. 17, No. 10, pp. 724-726, Oct. 2007.

[14] A. Hajimiri and T. H. Lee, "Design issues in CMOS differential LC oscillators," IEEE J. of Solid-State Circuits, pp. 717-724, May 1999.

[15] T. Lagutere, J. M. Pailot, and H. Guegnaud, "Method to design low noise differential CMOS VCOs without tail current source", Int. J. of Electronics and Comm., Vol. 60, No. 2, pp. 172- 
$178,2006$.

[16] S. Levantino et al., "Frequency dependence on bias current in 5-GHz CMOS VCOs: impact on tuning range and flicker noise upconversion," IEEE J. of Solid-State Circuits, Vol. 37, No. 8, pp. 1003-1011, Aug. 2002.

[17] E. Hegazi, H. Sjoland, and A. A. Abidi, "A filtering technique to lower LC oscillator phase noise", IEEE J. of Solid-State Circuits, Vol. 36, No. 12, pp. 1921-2930, Dec. 2001.

[18] A. Jeng and C. G. Sodini, "The impact of device type and sizing on phase noise mechanism", IEEE J. of Solid-State Circuits, Vol. 40, No. 2, pp. 360369, Feb. 2005.

[19] B. Soltanian and P. Kinget, "AM-FM conversion by the active devices in MOS LC-VCOs and its effect on the optimal amplitude", IEEE RFIC Symp., pp. 104-108, 2006.

[20] O. Casha, I. Grech, J. Micallef, and E. Gatt, "Design considerations and device selection in the implementation of low phase noise LC-VCOs," IEEE ISCAS, pp. 376-379, May, 2005.

[21] H. Wu, and A. Hajimiri, "Silicon-based distributed voltage-controlled oscillators," IEEE J. of SolidState Circuits, Vol. 36, No. 3, pp. 493-502, Mar. 2001.

[22] P. Andreani, and S. Mattisson, "On the use of MOS varactors in RF VCO's," IEEE J. of Solid-State Circuits, Vol. 35, No. 6, pp. 905-910, Jun. 2000.

[23] S. L. Jang, C. C. Liu, M. H. Suchen, et al., "An eight-phase CMOS voltage controlled oscillator," Microwave and Optical Tech. Lett., Vol. 51, No. 5, pp. 1225-1228, May 2009.

[24] T. Shibasaki, et al., "18-GHz clock distribution using a coupled VCO array," IEICE Trans. Electron., Vol. E90-C, No. 4, pp. 811-823, Apr. 2007.

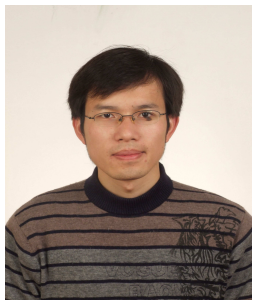

Changchun Zhang received the B.S. and M.S. degrees in communication engineering in Guilin University of Electronic Technology (GUET), Guilin, China, in 2003, and 2006, respectively, and $\mathrm{Ph} . \mathrm{D}$. degree in electrical engineering in Southeast University, Nanjing, China, in 2010. Then, he worked as a post-doc researcher at Ewha Womans University, Seoul, South Korea. In December 2010, he joined Nanjing University of Posts and Telecommunications, Nanjing, China. His current research interests include IC designs on RFID transceivers and wireline transceivers.

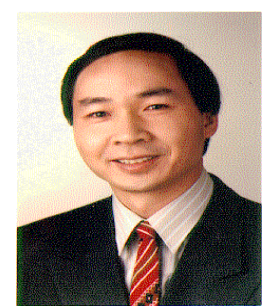

Zhigong Wang was born in Henan, China. He received the M.-Eng. degree in radio engineering from Nanjing Institute of Technology (now, Southeast University), Nanjing, China, in 1981, and the Dr.-Ing. degree in electronic engineering from RuhrUniversity Bochum, Germany, in 1990. From 1977 to 1981, he worked on radio communication techniques and computer aided circuit designs in Nanjing Institute of Technology. During 1982-1984 he worked as a lecturer on semiconductor circuit techniques in Tongji-University, Shanghai. From 1985 to 1990, he worked on high-speed silicon bipolar circuit designs for multi-gigabit/s optic fiber communication in Ruhr-University Bochum, Germany. From Oct. 1990 to Sept. 1997, he has been with Fraunhofer-Institute of Applied Solid State Physics, Freiburg, Germany, working on high-speed GaAs ICs for optic-fiber data transmission and MMICs. Since Oct. 1997, he is full professor of Southeast University, Nanjing, China. He is the author or co-author of 20 books and more than $320 \mathrm{SCI} / \mathrm{EI}$-cited papers, and inventor of 25 patents of China, Germany, Europe, USA, and Japan. He is Senior Member of IEEE since 1993, Chairman of the Advisory Committee of Electrical and Electronical Basic Courses of Chinese Universities. He is Guest Professor of more than 20 universities of China, Canada, and Australian. Recently, he is involving in IC design for optic-fiber transmission systems, for RF wireless, microwave, and millimeter-wave applications, and in micro-electronic systems for bio-medical applications. 


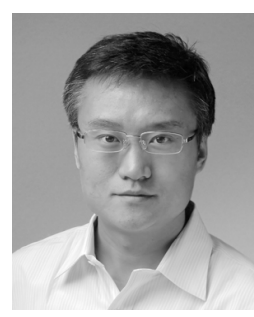

Yan Zhao received the M.S. degree in microwave technology from Northwestern Polytechnical University (NWPU) in 2002. From 2002 to 2005, he was with ZTE Corporation where he worked on mm-wave LMDS system design and WCDMA RF unit design. In 2009, he received Ph.D. in circuit and system from Southeast University (SEU), China. Since 2009, he is with the Institute for High-frequency and Communication Technology (IHCT) at the University of Wuppertal, Germany, and researching on mm-wave and terahertz circuit design.

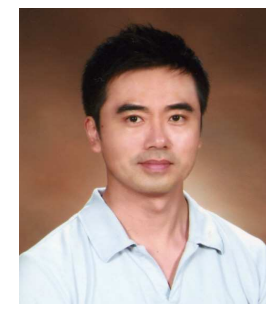

Sung Min Park received the B.S. degree in electrical and electronic engineering from KAIST, Korea in 1993. He received the M.S. degree in electrical engineering from University College London, UK in 1994 and the $\mathrm{Ph} . \mathrm{D}$. degree in electrical and electronic engineering from Imperial College of Science, Technology and Medicine, UK in May 2000. In 2004, he joined the faculty of the department of electronics engineering at Ewha Womans University, Seoul, Korea, where he is currently an associate professor. His research interests include high-speed analog/digital integrated circuit designs in sub-micron CMOS and SiGe HBT technologies for the applications of optical interconnects, silicon photonics, and RF communications. He has served a number of international conferences as a technical program committee, including ISSCC (20042009) and A-SSCC (2007-2008). 